\title{
Structural-temporal approach for dynamic strength characterization of gabbro-diabase
}

\author{
Y. Petrov ${ }^{1,2}$, A. Bragov ${ }^{3}$, A. Evstifeev ${ }^{1}$, and E. Cadoni ${ }^{4}$ \\ ${ }^{1}$ Saint Petersburg State University, 199034 St. Petersburg, Russia \\ 2 IPME RAS, Extreme States Dynamics Department, 199178 St. Petersburg, Russia \\ ${ }^{3}$ Lobachevski State University, 603950 Nizhny Novgorod, Russia \\ ${ }^{4}$ DynaMat Laboratory, University of Applied Sciences of Southern Switzerland, 6952 Canobbio, Switzerland
}

\begin{abstract}
This paper presents some of the results of dynamic compression, splitting and direct-tensile tests of gabbro-diabase using two split Hopkinson bar apparatus. The results of laboratory experiments on specimens without and with notch for a wide range of loading parameters are given. Strength and deformation characteristics of gabbro-diabase were determined experimentally at high strain rates up to $10^{3} \mathrm{~s}^{-1}$. Mechanical characteristics were found to be strongly depended on the strain rate. A uniform interpretation of the rate effects of fracture of the tested gabbro-diabase is given on the basis of structuraltemporal approach based on a set of fixed material constants. It is shown that temporal dependences of both the compressive and tensile strengths of the tested material can be effectively calculated using the incubation time fracture criterion.
\end{abstract}

\section{Introduction}

The dynamic characterization of rocks under high loading rates is fundamental to understand the material behaviour in case of dynamic events. The dynamic mechanical properties of rocks can be very different from those exhibited in quasi-static conditions. Specific investigations in such dynamic ranges appear necessary to correctly understand their behaviour under intermediate and high strain rate conditions. These data are necessary and require experimental techniques able to induce on the rock materials state of loading reproducing the actual dynamic condition. Also must be a simple criterion for a wide range of parameters of loadings.

The dynamic characterization has been carried out by means of traditional Split Hopkinson Pressure Bar (SHPB) and a Split Hopkinson Tensile Bar (SHTB) placed respectively at the Laboratory of Dynamic Investigation of Materials of Nizhny Novgorod and at the DynaMat Laboratory of the University of Applied Sciences of Southern Switzerland. These equipment are briefly described and the results of dynamic tension/compression tests at different high strain-rates on gabbro-diabase are discussed.

Thus, the objective of this work is the comparison between both tensile and compressive dynamic and quasistatic tests, and analysis obtained results with incubation time approach.

\section{Materials}

Gabbro is a dark, coarse-grained, intrusive igneous rock chemically equivalent to basalt. It is a plutonic rock, formed when molten magma is trapped beneath the Earth's surface and cools into a crystalline mass.
Gabbro is generally coarse grained, with crystals in the size range of $1 \mathrm{~mm}$ or greater. Finer grained equivalents of gabbro are called diabase, although the vernacular term "microgabbro" is often used when extra descriptiveness is desired. Gabbro may be extremely coarse grained to pegmatitic, and some pyroxene-plagioclase cumulates are essentially coarse grained gabbro, although these may exhibit acicular crystal habits.

Gabbro is usually equigranular in texture, although it may be porphyritic at times, especially when plagioclase oikocrysts have grown earlier than the groundmass minerals.

Gabbro - one of the most common intrusive rocks. This breed is composed of plagioclase and clinopyroxene in roughly equal proportions. Gabbro-diabase rock is the basic structure, with a massive texture, fine-medium grained, without cavities and irregularities uniformly painted in dark gray. Test specimens were taken from Drugoretskoe field (Republic of Karelia). Material properties are given in Table 1.

\section{Experimental and analytical details}

Static and dynamic loading tests have been conducted using different experimental set-ups on cylindrical rock specimens.

\subsection{Dynamic compression and splitting tests}

Dynamic compressive and splitting tests were performed at the Laboratory of Dynamic Investigation of Materials in Nizhny Novgorod by means of a SHPB shown in Fig. 1.

The experimental set-up consists of a compact gas gun (1), incident (2) and transmitter (5) steel (or duralumin) pressure bars with the specimen (3) sandwiched between 
Table 1. Material properties.

\begin{tabular}{|l|l|}
\hline Young's modulus, MPa & 91550 \\
\hline Average Compressive strength, MPa & 215 \\
\hline Average Tension strength, MPa & 17,5 \\
\hline
\end{tabular}

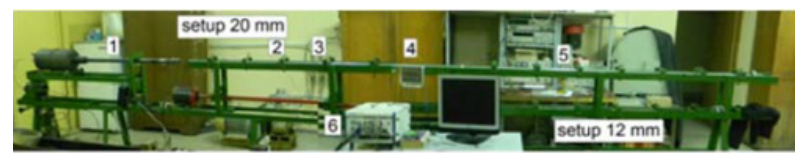

Figure 1. Experimental apparatus realizing SHPB for compressive and splitting tests.

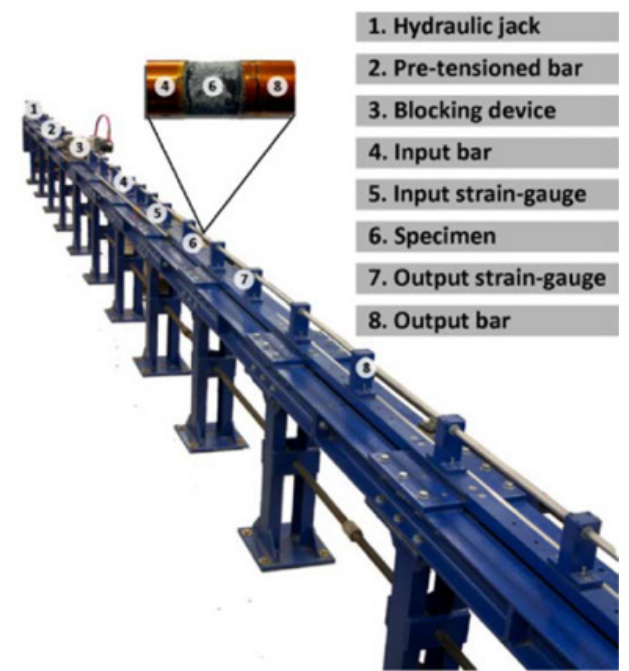

Figure 2. Split Hopkinson Tensile Bar used in this research.

them. Power supply and calibration of strain gauges was produced by an original scheme (4). In order to record the electrical signals from strain gauges, a multichannel digital oscilloscope (6) was used. Diameters of striker as well as pressure bar are $20 \mathrm{~mm}$. The incident bar length was $1 \mathrm{~m}$, whereas the length of transmitter bar was $3 \mathrm{~m}$ in order to provide correct registration of possible additional cycles of loading during the experiment [1].

Splitting test of disks, also called "Brazilian tests", is one of the available methods to measure the tensile strength of brittle materials [2]. Due to the stress-state of the disk, the failure is caused by tension when tensile stresses reach the tensile strength of the material on the diametric loading plane.

\subsection{Dynamic tensile tests}

The high strain-rate tests in tension were performed using a Split Hopkinson Tensile Bar (SHTB) [3], that consists of two circular aluminium bars, called input and output bars, having a length of 3 and $6 \mathrm{~m}$ respectively, with a diameter of $20 \mathrm{~mm}$ to which the specimen is glued on using a bicomponent epoxy resin (see Fig. 2).

The input bar is connected with an high strength steel pre-tensioned bar $6 \mathrm{~m}$ in length, used as pulse generator with a diameter of $12 \mathrm{~mm}$ in order to obtain the same acoustical impedance of the input bar so that the pulse reffection, due to the interface, is avoided.
With reference of Fig. 2 a test with the SHTB is performed as follows [4]:

1. Firstly, a hydraulic actuator (1), of maximum loading capacity of $600 \mathrm{kN}$, is pulling the pretension high strength steel bar (2). The pretension stored in this bar is resisted by the blocking device (3).

2. Secondly, the rupture of a fragile bolt in the blocking device, gives rise to a tensile mechanical pulse of 2,4 ms with linear loading rate during the rise time, propagating along the input (4) and the output (7) bars bringing to fracture the specimen (6).

As well as in the classical Hopkinson bar apparatus, the pulse propagates along the input bar with the elastic wave velocity equal to $\mathrm{C}_{0}$, and constant shape. When the incident pulse $\left(\varepsilon_{\mathrm{I}}\right)$ reaches the rock sample, it is partially reffected by it $\left(\varepsilon_{\mathrm{R}}\right)$. On the other hand, the portion of the pulse that is not reffected passes through the specimen $\left(\varepsilon_{\mathrm{T}}\right)$ and propagates into the output bar.

\subsection{The concept of incubation time}

Considering phenomena such as brittle failure of a defect free specimen, fracture of specimen with macro-defect (crack), we deal with strength properties of the materials. These very different processes exhibit some important common features. In the case of slowly applied load there exists the threshold value of load amplitude. In the case of very short durations the strength characteristics of materials are considerably different from those obtained in the case of quasi-static testing. This can be explained by the circumstance that the time of loading is of the same order as the typical time of certain processes on the micro-level. The above-mentioned time can be described by virtue of the material constant and will be referred to as "incubation time". An efficient criterion for the analysis of brittle fracture for defect-free material can be formulated in the following form [5-8]:

$$
\frac{1}{\tau} \int_{t-\tau}^{t} \sigma(s) d s \leq \sigma_{s t}
$$

where $\tau$ is the incubation time, $\sigma_{s t}$ is a tensile strength for quasi-static loading and $\sigma(t)$ is the applied stress which for $t<0$ is supposed to be zero. The instant of fracture $t^{*}$ corresponds to the earliest realization of equality in Eq. (1) or, in the general case, violation of this condition.

For our tasks (uniaxial extension) take stress $\sigma(t)$ in this form

$$
\sigma(i)=\dot{\sigma} I I I(l)
$$

where $H(t)$ is a Heaviside function, $\dot{\sigma}$ is a Stress rate (supposed to be constant).

Here we will use a generalization of critical condition (1) based on taking into account two major factors: level (amplitude) and rate of local loading of the continuum. The corresponding criterion is given by following condition [7].

$$
\frac{1}{\tau_{\alpha}} \int_{t-\tau_{\alpha}}^{t}\left[\frac{\sigma(s)}{\sigma_{s t}}\right]^{\alpha} d s \leq 1
$$




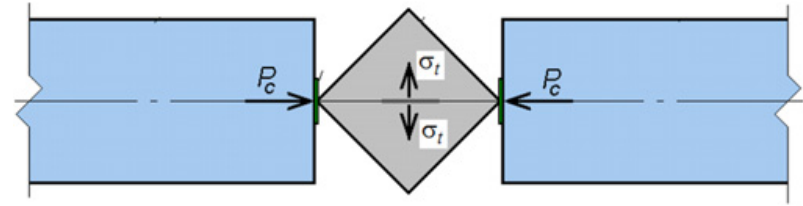

Figure 3. Experimental scheme for splitting tests.

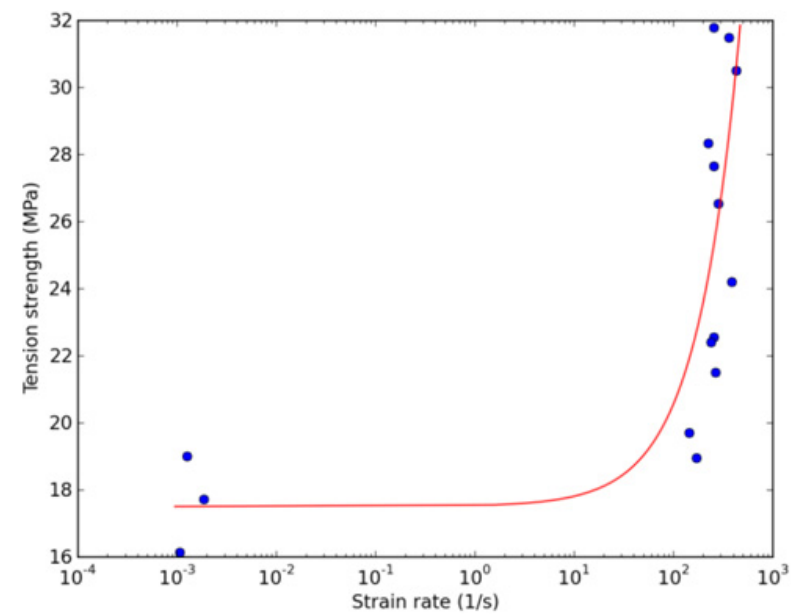

Figure 4. Tension strength.

where $\tau_{\alpha}$ is the incubation time. Incubation time characterises sensitivity of material to rate of loading at the given point of continuum. The parameter $\alpha$ characterises sensitivity to the level (or amplitude) of local stress. For most of the solid continua $\alpha>1$. The parameter $\sigma_{s t}$ is tensile strength for quasi-static loading and $\sigma(t)$ is the local stress which for $t<0$ is supposed to be zero like in the first case.

The general concept of incubation time works in both compressive and tensile cases, but the set material constants must be determined for compression and tension separately.

\section{Results}

The strain rate of the test is dependent on two variables: the behaviour of the material and the loading of the prestressed bar. A constancy of the preload has been chosen in order to verify the response of the rock specimen under the same loading condition.

\subsection{Compression and splitting tests}

Experiments for tension was completed like a model "Brazilian tests", but samples were in the form of rectangular parallelepipeds section $20 \times 20 \mathrm{~mm}$ with length 10, 20, $30 \mathrm{~mm}$. Experimental schemes on Fig. 3.

For tensile stress formula in the form

$$
\sigma_{t}=0.5187 \frac{P_{c}}{b h}
$$

where $\mathrm{b}$ and $\mathrm{h}$ - dimensions of the sample, $\mathrm{P}_{\mathrm{c}}-$ longitudinal force in the gauging rods, determined by indications on the support rod. The maximum value of the tensile stress is the required tensile strength of stone.

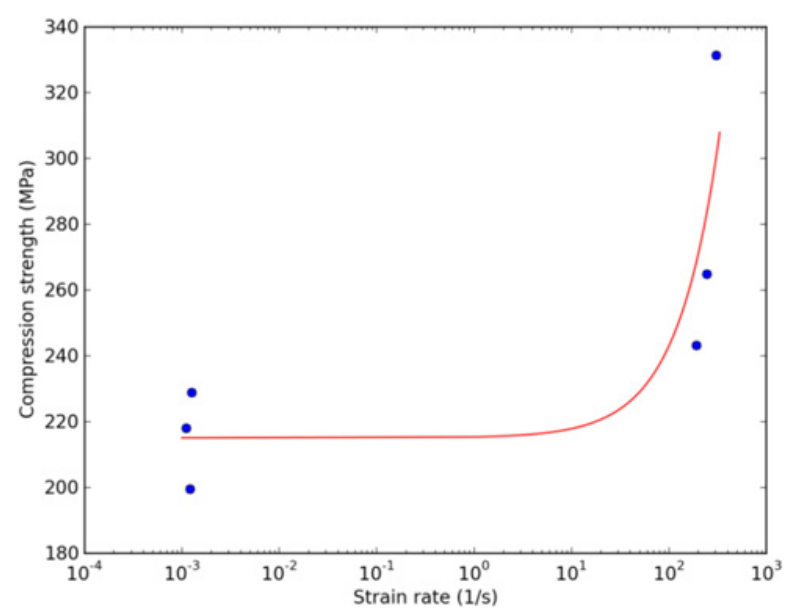

Figure 5. Compression strength.

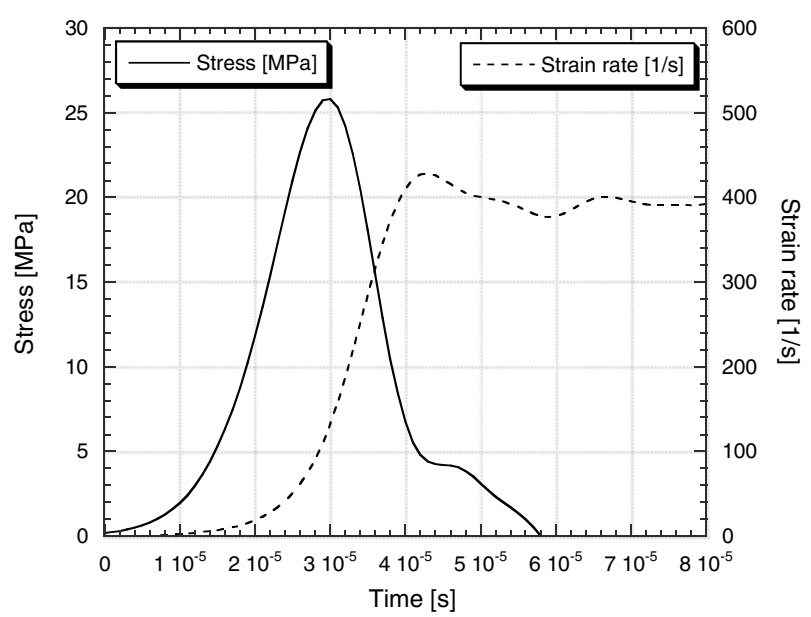

Figure 6. Tension strength and strain rate versus time.

Table 2. Dynamic tensile results ( $\dot{\sigma}=900 \mathrm{GPa} / \mathrm{s})$.

\begin{tabular}{|c|c|c|c|c|c|}
\hline Test & $\begin{array}{c}\sigma_{\max } \\
{[\mathrm{MPa}]}\end{array}$ & $\begin{array}{c}\varepsilon_{\mathrm{u}} \\
{[\% 0]}\end{array}$ & $\begin{array}{c}\text { Strain rate } \\
{\left[\mathrm{s}^{-1}\right]}\end{array}$ & $\begin{array}{c}\mathrm{W}_{\max } \\
{\left[\mathrm{J} / \mathrm{m}^{2}\right]}\end{array}$ & $\begin{array}{c}\mathrm{G}_{\mathrm{F}} \\
{\left[\mathrm{J} / \mathrm{m}^{2}\right]}\end{array}$ \\
\hline 004 & 28.36 & 1.09 & 54 & 936 & 531 \\
\hline 005 & 29.05 & 0.77 & 71 & 921 & 621 \\
\hline 006 & 29.05 & 0.82 & 73 & 935 & 634 \\
\hline Average & 28.82 & 0.89 & 66 & 931 & 595 \\
(st. dev) & $(0.4)$ & $(0.17)$ & $(10)$ & $(8)$ & $(56)$ \\
\hline
\end{tabular}

On Fig. 4 shows the effect of the dynamic tensile strength. Points show the experimental data. Curves show the theoretical line constructed by the formula (1) with parameters $\tau=66 \mu \mathrm{s}, \sigma_{\mathrm{st}}=17.5 \mathrm{MPa}$. We can see good compliance theoretical approach and experimental data. Parameter $\sigma_{\mathrm{st}}-$ static tensile stress. It is determines the horizontal section on theoretical curve. Parameter $\tau$ - can move theoretical curve to the right or to the left. Theoretical curve was built as a middle line of experimental data.

For compression tests were carried out standard experiments. On Fig. 5 shows the effect of the dynamic compression strength. Points show the experimental data. Curves show the theoretical line constructed by the formula (1) with parameters $\tau=6 \mu \mathrm{s}, \sigma_{\mathrm{st}}=215 \mathrm{MPa}$. 
Table 3. Dynamic tensile results $(\dot{\sigma}=1.5 \mathrm{TPa} / \mathrm{s})$.

\begin{tabular}{|c|c|c|c|c|c|}
\hline test & $\begin{array}{c}\sigma_{\max } \\
{[\mathrm{MPa}]}\end{array}$ & $\begin{array}{c}\varepsilon_{\mathrm{u}} \\
{[\% 0]}\end{array}$ & $\begin{array}{c}\text { Strain rate } \\
{\left[\mathrm{s}^{-1}\right]}\end{array}$ & $\begin{array}{c}\mathrm{W}_{\max } \\
{\left[\mathrm{J} / \mathrm{m}^{2}\right]}\end{array}$ & $\begin{array}{c}\mathrm{G}_{\mathrm{F}} \\
{\left[\mathrm{J} / \mathrm{m}^{2}\right]}\end{array}$ \\
\hline 007 & 29.01 & 0.826 & 107 & 1472 & 1176 \\
\hline 008 & 25.82 & 0.679 & 132 & 1602 & 1326 \\
\hline 010 & 23.32 & 1.068 & 163 & 1175 & 911 \\
\hline Average & 26.05 & 0.858 & 134 & 1416 & 1138 \\
(st. dev) & $(0.3)$ & $(0.19)$ & $(28)$ & $(219)$ & $(210)$ \\
\hline
\end{tabular}

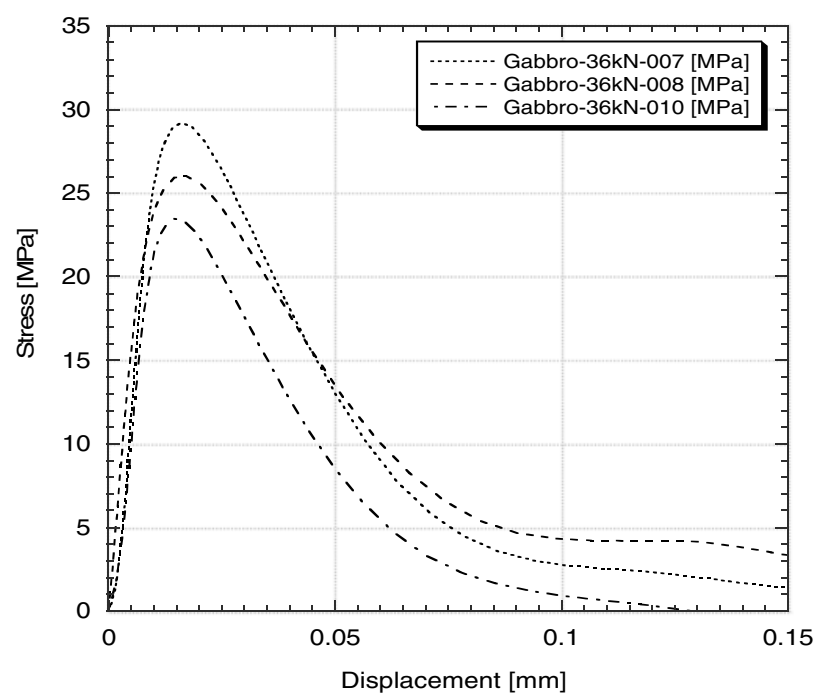

Figure 7. Stress vs. displacement curves in tension $\dot{\sigma}=$ $1.5 \mathrm{TPa} / \mathrm{s})$.

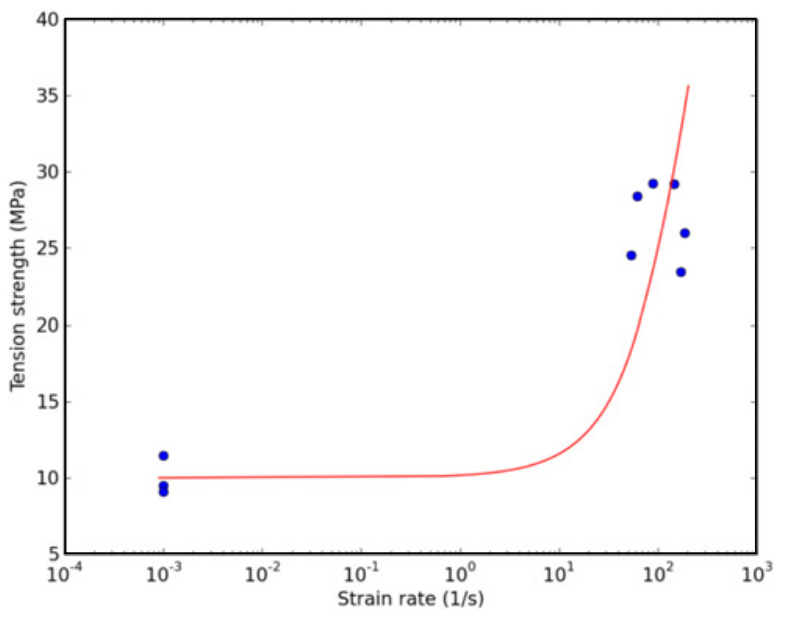

Figure 8. Tension strength.

We can see also good compliance theoretical approach and experimental data.

\subsection{Tensile tests}

Direct dynamic tensile tests on the gabbro-diabase were carried out at two strain-rates. The tests were obtained imposing $18 \mathrm{kN}$ and $36 \mathrm{kN}$ as pre-load in the pre-tensioned bar obtaining an average loading rate of $900 \mathrm{GPa} / \mathrm{s}$ and $1.59 \mathrm{TPa} / \mathrm{s}$, respectively. As well known for brittle materials the strain rate changes during the test as shown in Fig. 6 where the stress and the strain rate in function of time are depicted. As proposed in [9] the conventional strain rate can be read in the figure such as $132 \mathrm{~s}^{-1}$.

In Tables 2 and 3 are reported the results of the dynamic direct tensile tests.

In Fig. 7 the stress versus displacement curves are depicted for the higher stress rate.

On Fig. 8 shows the effect of the dynamic tensile strength. The specimens were notched. Points show the experimental data. Curves show the theoretical line constructed by the formula (1) with parameters $\tau=3.4 \mu \mathrm{s}$, $\sigma_{\mathrm{st}}=10 \mathrm{MPa}$. We can see good compliance theoretical approach and experimental data.

\section{Conclusion}

Dynamic tension, indirect and direct tensile tests of a gabbro-diabase were conducted using two set ups as Split Hopkinson Pressure Bar and Split Hopkinson Tensile Bar.

Rock tensile and compression strength is strain rate dependent. It has been veriffed that also for the gabbro-diabase this strength increases with the strain rate increasing within the wide strain rate range. The results show non-linear relationships between strength and strain rates.

It is shown that the mechanical characterization of tested rock can be effectively performed using two experimental setups.

A uniform interpretation of the rate effects of fracture of the tested gabbro-diabase is possible based on the structural-temporal approach and a set of fixed material constants. It is found that temporal dependences of both the compressive and tensile strengths of the tested material can be effectively predicted on the basis the incubation time fracture criterion.

Authors acknowledge Russian Foundation for Basic Research (14-01-00814), the program N25 of the Presidium of the Russian Academy of Sciences, FP7 EU MARIE CURIE TAMER (No.610547), and the Saint-Petersburg State University (6.38.243.2014) for the support.

A special acknowledgement goes to the Scientific \& Technological Cooperation Programme Switzerland-Russia for the financial support of the Utilisation of Specific Infrastructure Project called "Dynamic fracture of rocks under high rate mechanical and electric breakdown induced loads" between DynaMat Laboratory of the University of Applied Sciences of Southern Switzerland and the St. Petersburg State University.

\section{References}

[1] J. Rodriguez, C. Navarro, V. Sanchez-Galvez, J. Phys. IV France, 04 (1994) 101.

[2] Bragov, A.K. Lomunov, I.V. Sergeichev, J. Appl. Mech. Tech. Phys. 42 (2001).

[3] E. Cadoni. Rock Mech. Rock Eng. 43 (2010) 667-676. 
[4] A. Caverzan, E. Cadoni, M. di Prisco, Int. J. Impact Eng. 45 (2012), 28-38.

[5] Y. V. Petrov, and N. F. Morozov, J Appl. Mech. 61, 710-712 (1994).

[6] Y. V. Petrov, N. F. Morozov, V. I. Smirnov, Fatigue Fract Engng Mater Struct. 26, 363-372 (2003).
[7] A. Evstifeev, E. Cadoni, Y. Petrov, EPJ WoC 26, 01041 (2012).

[8] Y. Petrov, I. Smirnov, A. Evstifeev, N. Selyutina, Frattura ed Integrità Strutturale 24, 112-118 (2013).

[9] E. Cadoni, A. Meda, G. Plizzari. Mat. Struct. 42(9), 1283-1294 (2009). 